\title{
BMJ Open Why do doctors seek peer support? A qualitative interview study
}

\author{
Ingrid Marie Taxt Horne (D) , ${ }^{1}$ Frode Veggeland, ${ }^{2}$ Fredrik Bååthe,,${ }^{3,4}$ \\ Karin Isaksson $\mathrm{R}^{4}$
}

To cite: Horne IMT, Veggeland F, Bååthe $\mathrm{F}$, et al. Why do doctors seek peer support? A qualitative interview study. BMJ Open 2021;11:e048732. doi:10.1136/ bmjopen-2021-048732

- Prepublication history and additional supplemental material for this paper are available online. To view these files, please visit the journal online (http://dx.doi.org/10.1136/ bmjopen-2021-048732).

Received 05 January 2021 Accepted 01 October 2021

Check for updates

(C) Author(s) (or their employer(s)) 2021. Re-use permitted under CC BY-NC. No commercial re-use. See rights and permissions. Published by BMJ.

${ }^{1}$ Modum Bad Research Institute, Vikersund, Norway

${ }^{2}$ Department of Health

Management and Health

Economics, University of 0slo, Oslo, Norway

${ }^{3}$ Sahlgrenska Academy, University of Gothenburg, The Institute of Health and Care Sciences, Gothenburg, Sweden

${ }^{4}$ The Institute for Studies of the Medical Profession, Legeforeningens

Forskningsinstitutt, Oslo, Norway

Correspondence to

Ingrid Marie Taxt Horne;

ingrid.horne@modum-bad.no

\section{ABSTRACT}

Objectives To understand how doctors reflect on when and why they seek help from an organised peer-support service.

Design Data were collected through audiotaped, qualitative, semi-structured interviews. The interviews were analysed with systematic text condensation.

Setting A peer-support service accessible to all doctors in Norway.

Participants Thirteen doctors were interviewed after attending a counselling service in fall 2018 . They were selected to represent variation in gender, demographics, and medical specialty. Doctors were excluded if the interview could not be held within 10 days after they had accessed peer support.

Results The doctors' perspectives and experiences of when and why they seek support and their expectations of the help they would receive are presented, and barriers to and facilitators of seeking support are discussed. Three categories of help-seeking behaviour were identified: (1) 'Concerned-looking for advice' describing help seeking in a strenuous situation with need for guidance; (2) 'Fear of not coping any longer' describing help seeking when struggling due to unreasonable stress and/or conflict in their lives; and (3) 'Looking for a way back or out' describing help seeking when out of work. Expectations to the help they would receive varied widely. Motivations for seeking help had more to do with factors enabling or restricting help-seeking than with the severity of symptoms.

Conclusions Many different situations lead doctors to seek peer support, and they have various expectations of the service as well as diverse needs, motivations and constraints to seeking peer support. Further research is warranted to investigate the impact of peer support and how to tailor the service to best suit doctors' specific needs.

\section{INTRODUCTION}

There is a growing awareness that reduced wellness among doctors negatively influences patient care. ${ }^{1-4}$ Studies have shown greater reluctance among doctors to seek medical care and psychological support compared with other professionals. ${ }^{56}$ To facilitate help seeking, peer-support schemes have been instituted in several countries. ${ }^{7-11}$ To ensure the usefulness of peer-support services, it is crucial to understand when and why doctors
Strengths and limitations of this study

This study provides new knowledge of how doctors reflect on seeking help at a peer-support service.

- In-depth qualitative interviews contributed rich material giving insights into doctors' subjective experiences and help-seeking practices.

- Through a selection of eligible doctors based on gender, age, specialty and demographics, a wide range of help-seeking behaviours emerged. There is a risk of bias through under-representation of those who declined to be interviewed, potentially because they were too exhausted to participate or reluctant to share private information. To counteract bias, analyses and generation of codes were carried out within an interdisciplinary team.

- Although this study was conducted in Norway, we suggest that the knowledge generated about categories of support-seeking behaviour can be of value to organisations providing counselling services for doctors as well as to employers in healthcare, doctors' associations and the community of doctors.

seek peer support and to know more about the factors that enable and restrict them from seeking help.

According to the 2017 Revised Declaration of Geneva: A Modern-Day Physician's Pledge, doctors are obliged to care for their health: 'I will attend to my own health, well-being and abilities in order to provide care of the highest standard. ${ }^{12}$ Nevertheless, there is a persistent gap between doctors' knowledge of diagnoses and necessary treatment and their willingness to seek professional healthcare for themselves when required. ${ }^{13-15}$ This reluctance is multifaceted..$^{5}$ The traditional image of doctors as self-sufficient, capable and altruistic $^{16}$ contributes to them working tirelessly and to a culture of self-treatment. However, in recent years, there has been an increasing awareness of the necessity for doctors to be able to enjoy work-life balance ${ }^{13}{ }^{17-19}$ and promote self-care. ${ }^{20-22}$ Changing attitudes toward doctors' needs might challenge the very idea of what a doctor should be even among doctors themselves. ${ }^{13}$ According to 
recent Scandinavian studies, there is growing concern among medical students and junior doctors about doctors' demanding work conditions and lack of worklife balance, limiting the possibilities for self-care. ${ }^{1623}$

Efforts are increasing to define the best management to help doctors cope with distress. As doctors are generally reluctant to seek healthcare, specific programmes have been instituted to prevent stress and mental illness in this population. For several years, readily accessible peersupport programmes have been provided in the USA, England, Denmark and Norway. ${ }^{84-26}$ One might expect doctors' threshold for pursuing these services to have lowered over the years, but the traditional endorsement of a doctor as self-sufficient, in control, and accomplished remains intact. ${ }^{16}$ Seeking help exposes vulnerability.

A few studies have examined the reasons why doctors seek peer support. ${ }^{1027}$ However, most of the research has been quantitative and focused on changes in symptom burden. Little is known about the beliefs and reflections of those who seek peer support. Interviewing doctors who have sought guidance from a peer-support service in Norway allowed for exploring these issues.

\section{AIM}

The aim of this study was to understand how doctors reflect on why and when they seek peer support, the kind of help they expect to receive and the factors that enable or restrict seeking this service.

\section{METHODS}

This study was explorative and interpretative. ${ }^{28}$ Qualitative modes of inquiry were employed to understand doctors' reflections on why and in which context they seek peer support and the factors that enable or restrict help-seeking.

\section{The peer-support service}

Villa Sana is a short-term counselling programme for doctors established by the Norwegian Medical Association (NMA). The aims are to enhance health and life quality, strengthen professional identity and prevent burnout. Two kinds of interventions are offered. One intervention is a single-day counselling session (6 hours) with a trained mental health professional, where the doctor's life situation and steps needed to handle it are discussed. This can include advice on seeking formal medical treatment including psychotherapy. The other type of intervention is a group-based, week-long course for 8-10 doctors that includes boarding, daily lectures, discussion and physical activity and an individual counselling session. Themes of the lectures are possibilities and restraints in working life, the individual's resources and personality, identity, communication, team work and prevention of burnout. The interventions are easily accessible (no referral needed and short waiting time). NMA covers all expenses, which enables doctors from all parts of Norway to attend.
Since the programme is defined as a preventive and not a clinical intervention, no medical records are kept, which ensures anonymity. ${ }^{10} 29$ The service is publicised on the homepage of the $\mathrm{NMA}^{30}$ and in the NMA journal.

If concerns arise regarding a help-seeking doctor's ability to treat patients or if ongoing suicidal plans are revealed, the counsellors, in cooperation with the doctor, initiate contact with a general practitioner (GP) and/or mental healthcare provider.

\section{Participants and sampling}

Qualitative in-depth interviews were conducted with doctors who sought a peer-support service in Norway, from August to December 2018. ${ }^{10}$ It appeared that saturation was being reached after about 10 interviews, and 13 interviews were conducted in total. This generated a rich dataset to analyse. Interviews were conducted by the first and last author, both medical doctors who have practiced as peer counsellors.

As doctors reach out for peer support at a vulnerable time in their lives it was important that they first received counselling, and that the research interview was subsequent to this. A voluntary, written consent was obtained from participants who were either recruited before the counselling by a mail request or by a direct request when they attended the counselling. As this is a highly confidential service, no record was made of those doctors who attended peer support but did not consent to take part in the study. 'Typical case sampling, ${ }^{28}$ drawn from demographic data, was applied. As the objective was to provide a wide distribution of characteristics of doctors seeking peer support, the sample is illustrative, not definitive. $^{28}$ Thirteen doctors (nine females; four males) were selected for interviews. Gender distribution ( $70 \%$ female, $30 \%$ male) and distribution between hospital specialties $(68 \%)$ and general practice (38\%) was approximately the same as among the 288 help-seeking doctors at the peer-support service in 2018. Participants came from different parts of the country and the age distribution was $25-70$ years (average age: 42 years; see table 1 ). As the intention was to capture the situation when seeking peer support, candidates were excluded when it was not possible to conduct the interview within 10 days after they had obtained peer support.

\begin{tabular}{lll}
\hline Table 1 & Participants' $(\mathrm{n}=13)$ background information & \\
\hline Gender & Male & 4 \\
& Female & 9 \\
Medical & Family medicine & 4 \\
specialty & Surgical specialties (gynaecology, & 3 \\
& $\begin{array}{l}\text { otolaryngology) } \\
\text { Laboratory medicine (biochemistry, }\end{array}$ & 2 \\
& radiology) & \\
& Psychiatry & 1 \\
& Internal medicine (geriatrics, paediatrics, & 3 \\
& internal medicine) & \\
\hline
\end{tabular}




\section{Patient and public involvement}

Patients were not involved in the design or planning of the study. A user-a doctor who had sought support from the peer counselling service-was involved in planning the study, writing the project description, and revising the present paper.

\section{Data collection and processing}

The semistructured interviews (1-1.5 hours) explored reasons why participants sought help, how they perceived peer counselling and impacts the counselling had on them. Please see online supplemental files for interview guide. The first author conducted all 13 interviews and the last author coconducted 9 of them, and they took place in locations feasible for all parties. Interviews were audiotaped and subsequently transcribed verbatim by a research assistant. Each interview was given a number and uploaded to a secure database; the identification code was stored separately. The interview data were complemented by observational field notes.

\section{Data analysis}

The interviews were analysed using Malterud's systematic text condensation. ${ }^{31}$ Descriptive and explorative analysis of the material followed four basic steps: (1) reading through the material to achieve an overall impression, from chaos to themes; (2) identifying and sorting meaning units, from themes to codes; (3) condensation, from codes to meanings; and (4) synthesising, from condensation to descriptions and concepts. ${ }^{31}$ The first and last author analysed the first few interviews in parallel and discussed the themes and codes together with the other coauthors until reaching a consensus. The first author analysed the remaining interviews independently. All the authors discussed data generation and concept development, resulting in the emergence of three categories of help-seeking behaviour. The interview texts were then reanalysed to produce rich portrayals of these three categories. The categories were assessed to determine whether they were sufficiently supported by data and then were revised again. The descriptions defining each category were then condensed, and they are presented with illustrating citations.

\section{RESULTS}

A wide spectrum of circumstances concerning doctors' help-seeking behaviour was analysed. The perspectives and experiences of when and why doctors seek support are presented in three categories constructed from the empirical material: (1) 'Concerned-looking for advice,' (2) 'Fear of not coping any longer,' and (3) 'Looking for a way back or out.' Rich descriptions of the categories follow. Further, the doctors' expectations of the help they would receive and the barriers to and facilitators of seeking support are presented.
Doctors' perspective and experiences of when and why they seek support

\section{Concerned-looking for advice}

In this category, doctors described obligations to perform both at work and at home, resulting in worries and lack of sleep. A feeling of uneasiness linked to the responsibility of being a doctor, such as fear of making mistakes, feeling incompetent or feelings of not being needed, was expressed. A demanding work situation was often accompanied by a challenging private situation.

Everyday life was perceived as strenuous, with energy depletion and a feeling of being behind schedule. While describing satisfactory coping with everyday life, 'warning signs,' such as crying at night, feeling a high level of stress over time, becoming more easily irritated, feeling blue and struggling to master work-life balance, were also experienced (table 2). This group of doctors was so exhausted physically and emotionally that some felt grateful to be able to stay home from work with a sick child or even expressed that they hoped for a fractured leg to claim a 'legitimate' reason to obtain sick leave. In addition, they could feel worries concerning their work, or be unable to enjoy their leisure time because they were constantly thinking about work (table 2 ).

I thought I would be able to enjoy my leisure time more than I do. I feel that I take work home with me every day, always something to think about, a patient you feel uncertain about. (Interview 6)

\section{Fear of not coping any longer}

Doctors in this category described unreasonable stress and/or conflict in their lives and going out of their way to accomplish what was expected of them both at home and at work (see table 2). Understaffed workplaces and expectations to work very long hours could lead to doctors being unable to take leave or sleeping very little. To cover for colleagues who were on vacation, some doctors overworked to a state of exhaustion. This exhaustion could even outlast their subsequent time off. In addition, serious conflict or power struggles with colleagues or management could cause isolation, loss of important work tasks or exclusion from taking part in reorganisational processes and meetings. Fear of not managing the next stretch of working life was expressed.

These situations led to concentration problems, sleeplessness, hopelessness and even depressive symptoms. Struggling to find words, feeling nauseous and a constant feeling of exhaustion were expressed (table 2). The simplest everyday tasks, such as calling in a patient from the waiting room or shopping, were difficult. The perceived inability to cope led to feelings of not being able to take control of the situation and that there was an endless list of duties both at work and at home forcing them to constantly rush to complete the next task. At times, there was an experience of everything coming to a halt. Doctors described difficulties in completing a train 


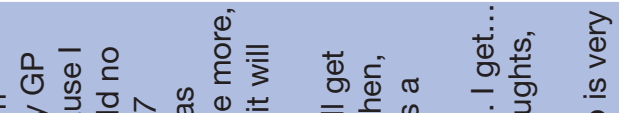

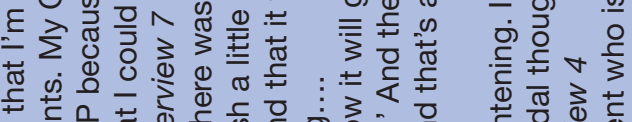

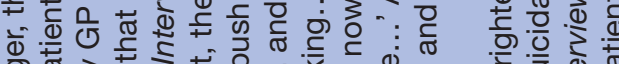
बृ $\begin{gathered}0 \\ 0\end{gathered}$ 흐의의

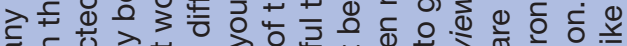
ब

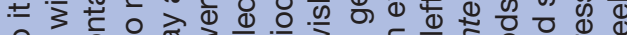

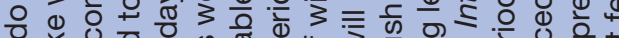

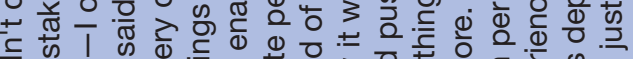

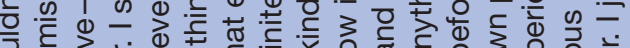
ठ

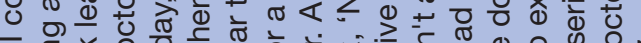

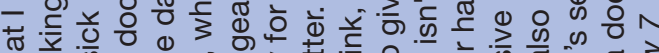
制

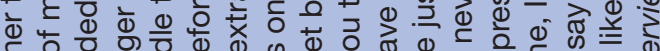

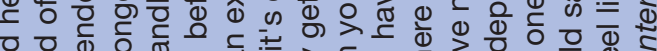
응 원 \pm का हो

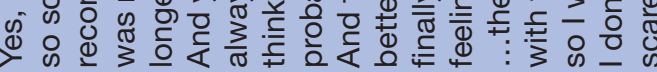

(i) 을효 入े

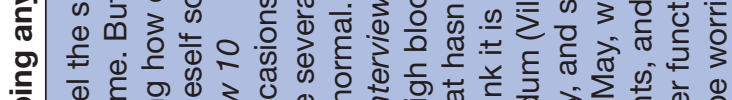

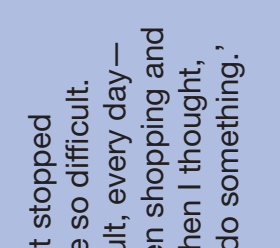

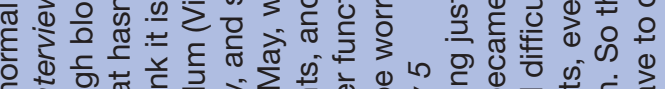
ब

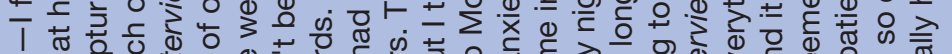

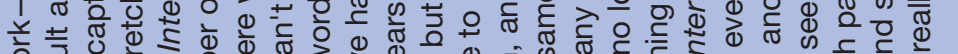

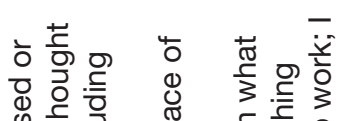

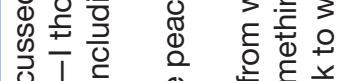

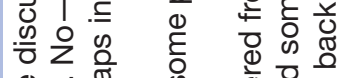
造语

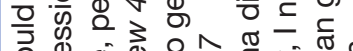
วิ ᄃ

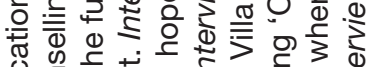

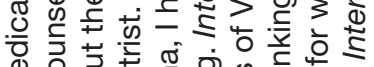

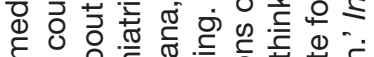
势

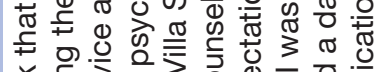

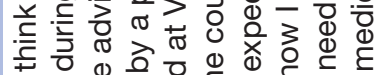

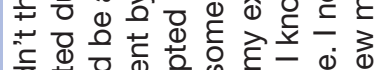

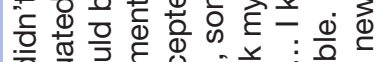
क् : उ. 난 1 का

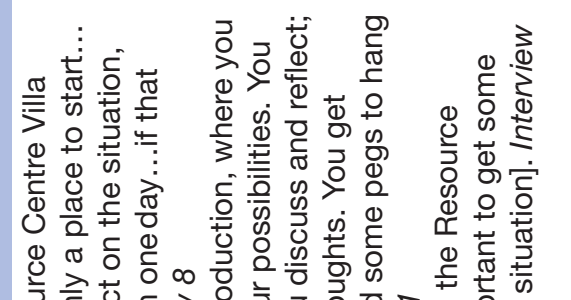

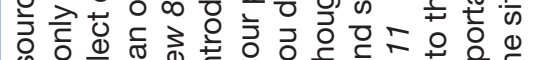

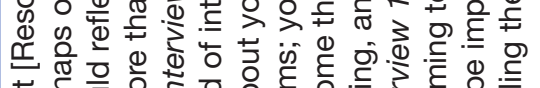

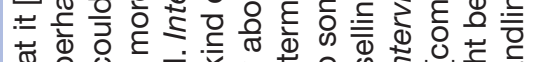

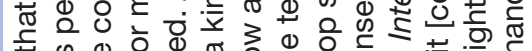
至舟

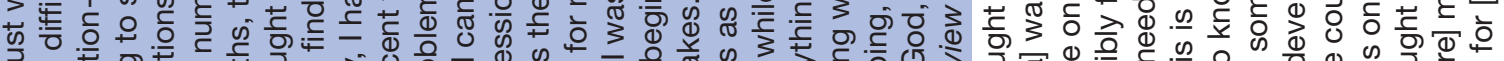

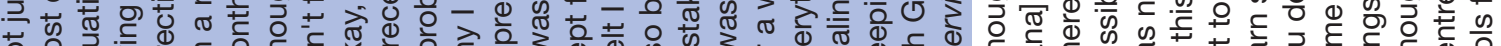

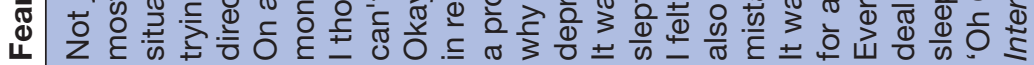

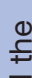
(2) ठ 하

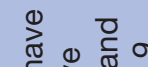

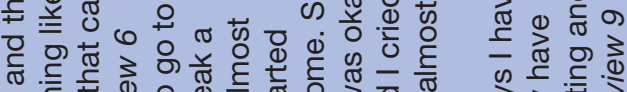
ब。

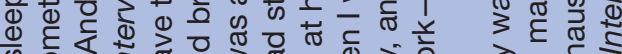
क प̄

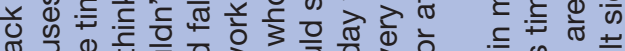

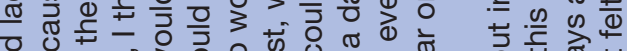

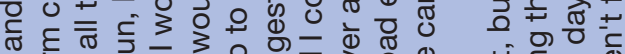

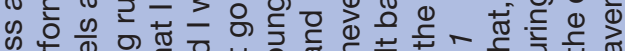
की क 茴 애

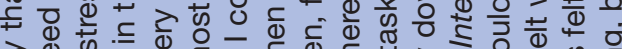
ते क क व

\section{동} 금 $\cong \stackrel{0}{ \pm}$

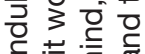

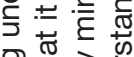

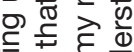

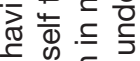

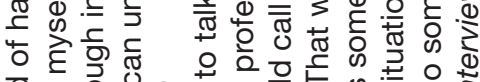

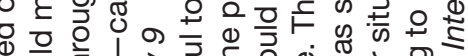

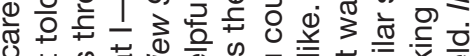

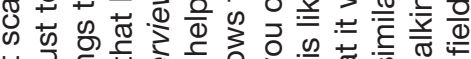
능.

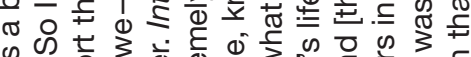

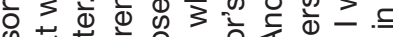

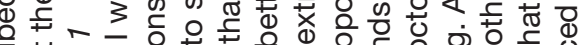

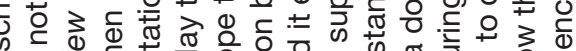

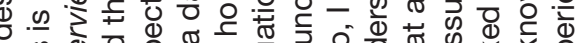

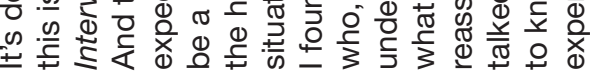

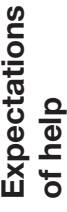


离

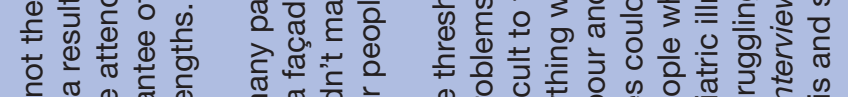

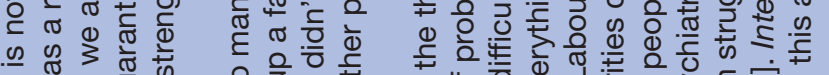

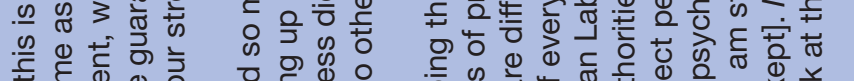

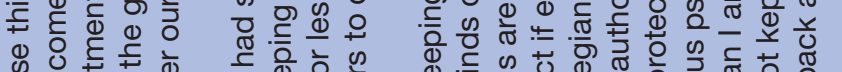

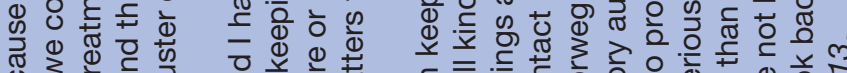
ه্

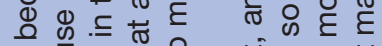
다에

政

Ф0

ᄃ

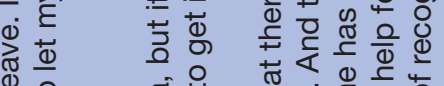

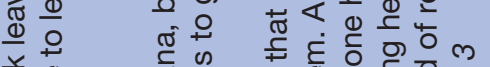

늘

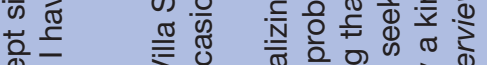

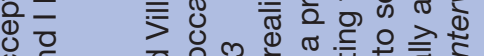

व 0 उ

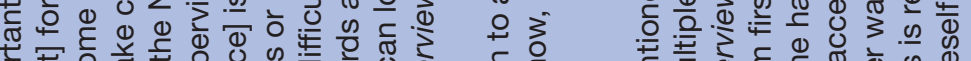

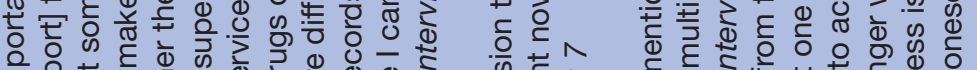

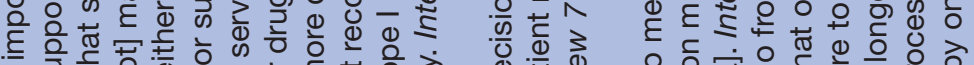

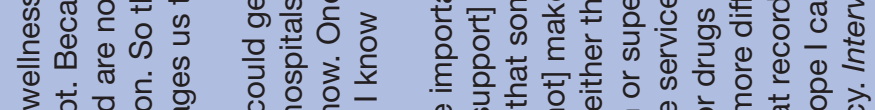

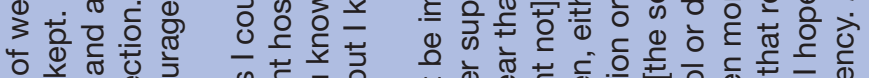

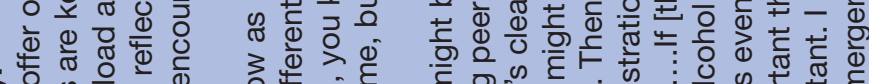

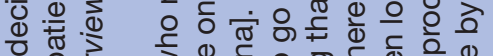

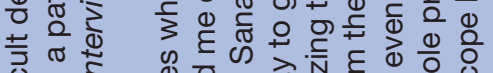

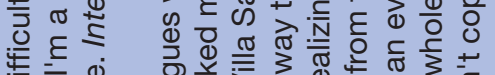

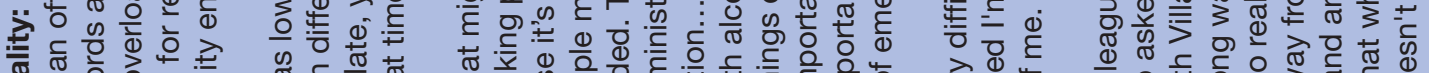
ప 는 일

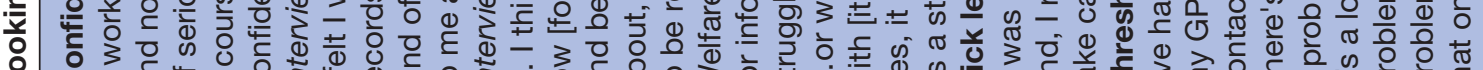

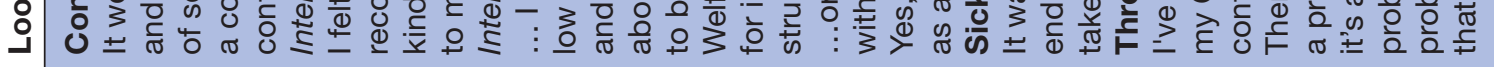

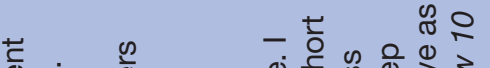

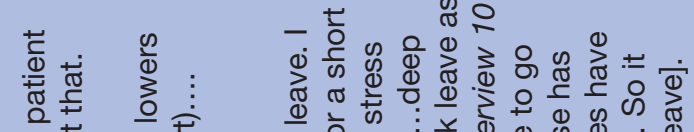

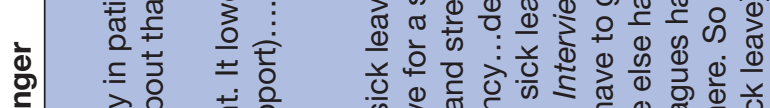

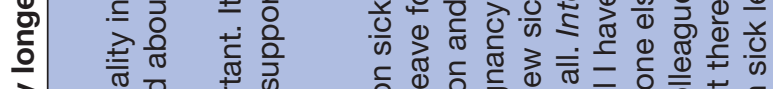

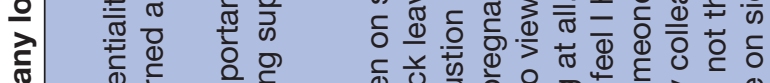

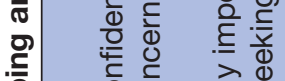

등

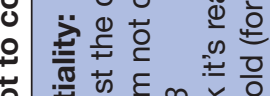

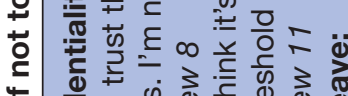

ه

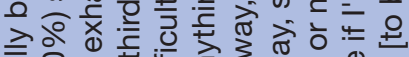

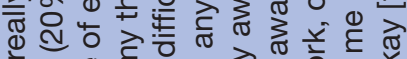

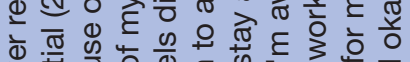

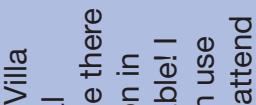

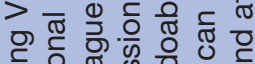

은

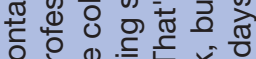

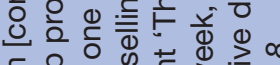

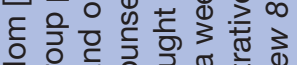

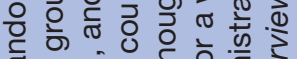

तั

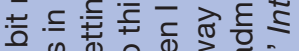

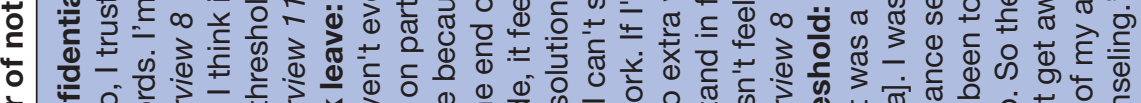

ఫ)

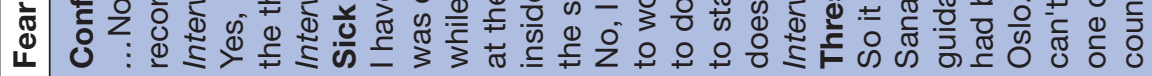

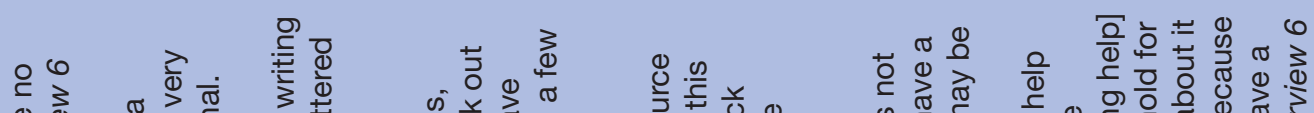

类

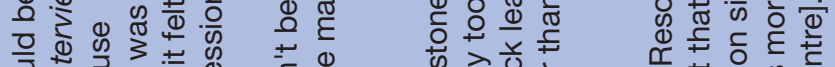

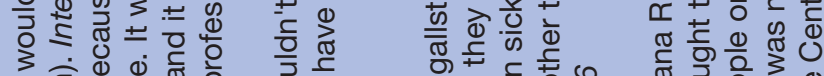

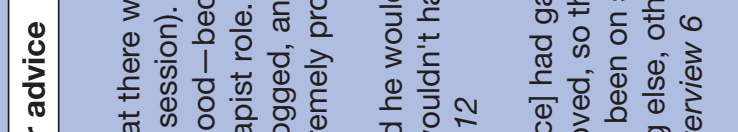

प 00 :

ल

๘઼

ब

产衣 至

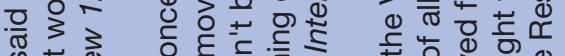

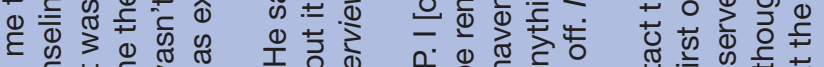

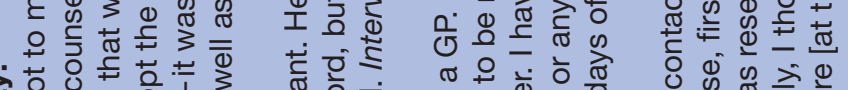

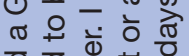

ठ용

प त ब

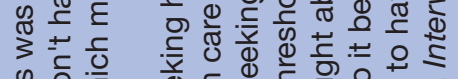

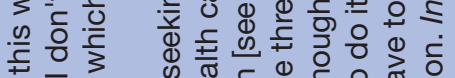

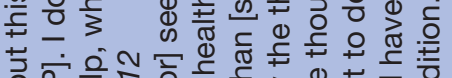

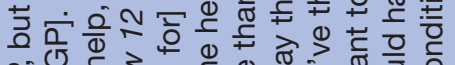

वें

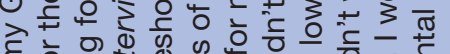

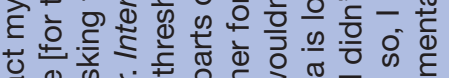

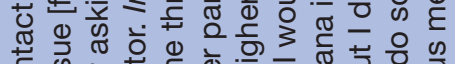

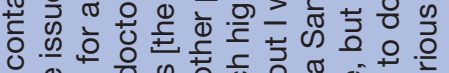

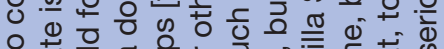

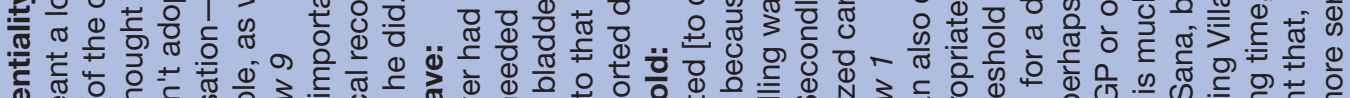
ब 는 $\varepsilon$ 믐

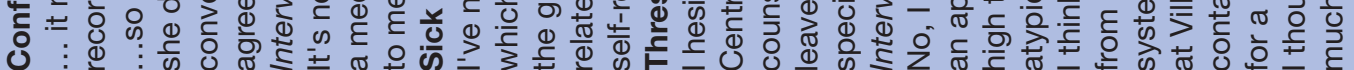


of thought, becoming anxious about the next patient and experiencing a reduced quality of life (table 2).

What's been such a dilemma for me is sick leave or no sick leave. And it has been through the last 3 years. (Interview 5)

\section{Looking for a way back or out}

Characteristics that fit into this category generally illustrate the significant pressure on doctors, such as long work hours and serious incidents (eg, patient suicide, murder threats or media exposure), often in combination with depression. These doctors had been diagnosed with either a somatic or psychiatric condition and were not working at the time of the interview. Often, they had hid serious symptoms while attempting to 'normalise' their condition. Some believed that they had to cope with the stress, as they assumed other colleagues did. However, finally, pain or reduced function prompted support seeking.

Sleep disturbances, anxiety, pain and depression were common. There were experiences of deterioration of memory and difficulties in performing simple practical tasks. Work had consumed all their energy and doctors described giving up exercise and becoming socially isolated. Self-harm and despair due to a situation not compatible with normal life or work activity led to serious suicidal thoughts (table 2). There was a fear of repercussions for needing accommodations at work. Some worked with a feeling of numbness. Doctors described being pushed to take on more patients than they felt they could handle and making mistakes resulting from lack of focus.

I think the worst experience I ever had was when I went on sick leave. It was the same feeling as giving up. I felt totally powerless. There were no options. (Interview 3)

\section{Doctors' expectations of the help they would receive}

Expectations to the help they would receive varied widely. Some doctors had limited clarity about what kind of help they could expect. Others did not really know what kind of help they needed (table 2). They all knew that the peer-support service is a doctor-specific service outside of the public healthcare system.

Doctors in the category 'Concerned-looking for advice' sought peer support primarily for guidance. It was important to meet a non-judgmental professional, outside of their own professional setting, who could provide advice and input (table 2). They expressed concerns that a more serious situation might arise if they did not act to cope with their issues in a better way. Some examples of expressed needs were hoping to receive advice, managing a work transition, seeking help to sort a situation with too many part-time jobs, or discussing if they should take advantage of their sabbatical leave to take care of their mental health.
Doctors in the category 'Fear of not coping any longer' considered seeking peer support as a place to start, hoping it could enhance their understanding of what the next step toward a better life should be. They sought advice or 'tools' for handling the current situation (table 2). Through counselling a need for referral to therapy could be revealed, and if so disappointment was expressed that the peer-support service did not offer treatment or referrals.

Although the doctors in the category 'Looking for a way back or out' had been in contact with the healthcare system, were diagnosed and were on sick leave or out of work due to their situation, they acknowledged a need for more help. There were hopes to access this through the counselling service, for example, through referral to a specialist (table 2). Ways to resume work, specific input about career changes and assessments concerning psychotropic drugs were among the presented needs. There was also a need for help to learn more about how to handle crises and trauma in private life and to handle the pain transferred from patients and patients' relatives.

\section{Barriers and facilitators}

As the empirical material revealed a wide range of opinions within and across the three categories concerning barriers and facilitators, see table 2, these findings are summarised here.

Regarding the importance of confidentiality in the peer-support service, participants' opinions differed. Some believed it was fundamental that the service be completely confidential so that no records could be accessed by colleagues or governing services. For others, it was not important whether a medical record existed or not (table 2). Some participants wished that there was a medical record, as this would have facilitated referral to further treatment. Others expressed that confidentiality promotes an important attitude in the counsellor about acting as a peer and not as a therapist (table 2). The confidential setting was seen as lowering the threshold to seek help, which was considered useful especially in reaching out to colleagues suffering from alcohol or drug abuse.

Sick leave was difficult to embrace. Interestingly, doctors who experienced a low threshold for seeking peer support were still reluctant to take sick leave when they felt unwell. The empirical data revealed an attitude towards sick leave as a practice that should be avoided if possible. They characterised leaving their work tasks as showing disloyalty to colleagues and patients even when sick leave is necessary (table 2). Those who were on sick leave or out of work described their struggle to keep up with work until they reached a tipping point (table 2). Not being able to work as a doctor was experienced as a loss of identity and described as a weakness, which was considered equivalent to 'giving up.'

Doctors who sought help as a preventive measure described this behaviour as 'atypical' of doctors (table 2). They had a low threshold for reaching out to the peersupport service-lower than for contacting the healthcare 
system. However, they had given a lot of thought before reaching out for peer support. Others considered the threshold to seek peer support as high and had struggled with distress for months to years before seeking help. Those who had previously sought help from the healthcare system due to the issue at hand opted for peer support as a supplement to regular treatment, not as a primary preventive measure. The doctors were aware of the services mainly due to a recommendation from their GP or a friend (see table 2).

\section{DISCUSSION}

This study reveals various reasons why and when doctors seek peer support. Three categories emerged from the rich empirical material: 'Concerned-looking for advice,' 'Fear of not coping any longer' and 'Looking for a way back or out.' Some of the doctors had sought support at a stage where it was possible to stop development towards ill health and burnout. Others had been clearly in need of support due to serious health or work issues that they were experiencing. However, why doctors turn to a peersupport service for help cannot be linked exclusively to the three categories presented above nor to the doctors' expectations of the help they would receive. This must be understood in light of experienced barriers to and facilitators of seeking peer support. This is further discussed below.

\section{Strengths and weaknesses}

An important strength of this study is that it promotes an increased understanding of how doctors reflect when seeking peer support by depicting information-rich, illuminative categories. The selection of eligible doctors based on gender, age, specialty, and demographics yielded descriptions of a wide range of help-seeking behaviours.

Arguably, 13 participants constitute a small sample size. However, Patton states that 'Qualitative inquiry typically focuses in depth on relatively small samples, even single cases $(n=1)$, selected for a quite specific purpose. ${ }^{, 28}$ In this rich empirical material, there was a sense of saturation after approximately 10 interviews and 3 more were added. Of course, there can be other experiences in other situations.

As there is no information about those who declined to participate in the study, the results could be biased. Possibly, doctors with severe exhaustion or who were very reluctant to share private information did not consent. However, it is important to emphasise that the interviewed doctors did share sensitive information that can create resonance and a sense of undeniability for the reader. ${ }^{32}$ Although this study was done in Norway, it dealt with common factors, such as doctors' reluctance to seek healthcare and a demanding work-life balance, ${ }^{13}{ }^{33}$ that have been documented in many studies in Western contexts. The phenomena described are, thus, general and relevant beyond the group of doctors interviewed in this study, as described by Wenger's theory of occupational community of practice. ${ }^{34}$ As such, the results have transferability to settings where doctors seek peer support. In light of the 'burnout epidemic' described in the USA and other Western countries, we need more qualitative knowledge of doctors' help-seeking.

The fact that the first and last authors are doctors themselves and have practiced as peer counsellors probably facilitated recruitment through credibility and also facilitated the interviews through firsthand knowledge of peer support. Nevertheless, bias may arise when investigating one's own organisation, ${ }^{28}$ through omitting relevant questions or modes of exploration, as this might be part of the researchers' tacit knowledge. This bias was counteracted by two of the coauthors having a non-clinical background, but extensive insight into the field of medical professionalism. The internal validity of the data were ensured by letting participants read the results section and approve citations. A user representative (a doctor who has sought peer support from this service) was involved in the planning of the study and project description and provided feedback on this paper.

\section{Barriers to and facilitators of seeking peer support Threshold}

The threshold for seeking peer support varied from low to high. Doctors identifying the threshold as low characterised this attitude as an 'atypical behaviour.' They had turned to peer support for guidance and did not express expectations of further treatment. Identifying the threshold as high was associated with severe symptoms and late help-seeking behaviour. Numerous factors contributed to a high threshold. In accordance with previous studies, participants unfavourably comparing themselves to colleagues who seemingly coped well with their challenging daily lives discouraged them from seeking help. ${ }^{35}{ }^{36}$ Concerns regarding confidentiality and fear of being labelled as unwell contributed to a higher threshold and were congruent with barriers identified in previous studies of why doctors avoid or postpone seeking medical care. ${ }^{6}{ }^{36}$ A recommendation from their GP or from a doctor friend who had attended the programme was a strong enabler of reaching out. In fact, all but one of the participants attended peer support after receiving a recommendation from a fellow doctor. It appears that the decision to seek peer support was regarded as difficult, although not as difficult as deciding to use healthcare services.

\section{Confidentiality}

Norwegian peer-support services are preventive and the programmes guarantee complete confidentiality. ${ }^{10}$ As the service is not referral-based, there is no preselection. This contributes to the wide spectrum of problems and needs found in this material. The lack of formal notes or records represents a double-edged sword. To some, confidentiality is a premise that enabled them to reach out to the service, lowering the 
threshold. Others noted the benefits of a formalised record, which could have provided the opportunity for referral to treatment. Yet other participants already had patient records in public healthcare, trusted that the records would be kept confidential, and therefore, expressed a neutral attitude towards the question of confidentiality. These findings reflect a potential dilemma between expectations (eg, referral to treatment) and the preventive nature of the peer-support service. ${ }^{37}$

Several participants emphasised the importance of keeping the services completely confidential to facilitate help seeking for those who struggle with drug or alcohol abuse or severe mental illness. Internationally, confidentiality in peer services for doctors is solved in a range of ways, from treatment programmes with medical records tailored for doctors as patients ${ }^{38} 39$ to peer-support programmes practicing confidentiality, but the latter have limited confidentiality to prevent harm to the doctor or patients. ${ }^{78}$ This places the Norwegian services in a position of emphasising confidentiality more than other peersupport services. In studies conducted in other countries, doctors suffering from burdens parallel to those of some participants in the present study were admitted to the hospital for treatment. ${ }^{3839}$

\section{Sick leave}

Numerous studies have documented doctors' reluctance to take sick leave when they need it. ${ }^{6640}$ Several factors contribute to this reluctance, but an underlying cause could be the need for a fundamental shift in perceived identity: a salesman does not become a customer on falling ill, whereas a doctor must step out of their professional role to become a patient. For many doctors, going to work while feeling unwell is considered an attribute; stories of colleagues battling heroically through the workday, despite fever or pain, are numerous. ${ }^{40}$ In this study, across all three categories, sick leave was not thought of as a viable option. Among doctors who were on long-term sick leave or out of work, inability to work as a doctor was voiced as a loss of identity. Indeed, some sought peer support as a supplement to public healthcare specifically in search of advice on ways to resume work as a doctor within a framework that supports work-home balance and mental health. Even though the peersupport service does not offer specific career guidance it can be a useful supplement to discuss career options. There seems to be a need for more flexible work hours, including the possibility of part-time work. Getting back to work can restore the previously mentioned sense of lost identity. Studies have shown that doctors demand of themselves to be 'fully capable.' However, this socialisation into a professional culture of altruism and invulnerability can make it challenging for doctors to take responsibility for their well-being. Young doctors now advocate an emerging new work identity, with a greater emphasis on self-care and work-home balance. ${ }^{13} 1623$

Since the late $1980 \mathrm{~s}$, concerns about doctors' reluctance to seek help for health issues have been reported. ${ }^{41}$ Despite increased awareness around this issue and the subsequent introduction of preventive peer-support programmes in several countries, this study found that all participants, to some degree, prioritised work at the cost of personal life and health. There are several reasons for this. For example, doctors find it challenging to assess their illness and carry out their work while feeling unwell because they prioritise their patients and colleagues. Younger doctors report fears of missing out on certain procedures and important learning as well as an element of competitive presenteeism. ${ }^{540}$ There is also a culture of self-treatment and seeking medical advice from doctors who are family related. ${ }^{42}{ }^{43}$ For many doctors, a personal mental struggle is perceived as a weakness; thus, help seeking can be stigmatised. To mitigate this, doctors sometimes search for more 'appropriate' motives to seek help when required. According to Giæver et al, doctors divide illness into 'respectable illness,' such as infections, stroke and cardiac infarction, and illness considered not legitimate enough to miss work, such as chronic and mental illnesses. ${ }^{40}$ Many of these considerations were apparent among participants in this study and explicitly contributed to the postponement of adequate help-seeking.

\section{Implications}

The empirical material in this study, as well as several previous studies, demonstrated doctors' tendencies towards symptom minimisation, self-medication and reluctance to seek support, especially for mental health issues. ${ }^{44} 645$ This underlines the pressing need for an organisational culture change in the medical profession to enable doctors to reach out, expose vulnerability and be human. Doctors should not consider help seeking as a sign of weakness or attempt to find 'somatic' reasons for missing work. It appears unreasonable that our society nourishes an understanding of 'the good physician' as altruistic and compassionate, yet simultaneously pushes doctors to adopt a judgmental attitude towards their own distress. This discussion should be of value to employers in healthcare, doctors' associations, the community of doctors and organisations providing counselling services for doctors in other countries.

\section{CONCLUSION}

This study identifies how help-seeking doctors reflect on a range of situations leading them to access a peersupport service and their expectations of the service. Despite comprehensive research on the risks of burnout and distress among doctors, and the institution of lowthreshold peer-support services, doctors hesitate to seek help. Why doctors seek help at a given time appears to be influenced more by their perceived threshold to reach out, their beliefs concerning confidentiality, and their 
attitudes towards sick leave than by symptom severity or worries about their condition.

Further research is warranted to understand doctors' experiences of the ability of peer support to meet their range of expectations. Thus, follow-up interviews are therefore planned.

Acknowledgements The authors wish to thank all doctors who supported this study by participating in the interviews.

Contributors IMTH and KIR designed the study, carried out the data collection and analysed, interpreted and drafted the paper. FV and FB participated in the study design and interpretation of data. All authors made critical revisions and contributed important intellectual content. IMTH is responsible for the overall content as the guarantor.

Funding This work was supported by the psychiatric institution Modum Bad and the Medical Aid and State Pension Arrangements for Doctors, both not-for-profit organisations. We confirm researcher independence from the funders and that all authors had full access to all data in the study and can take responsibility for the integrity of the data, accuracy of the data analyses, results and conclusions.

Competing interests None declared.

Patient consent for publication Not applicable.

Ethics approval The study obtained ethics approval from the Regional Ethics Committee (REC), Norway (REC ID number 2018/199).

Provenance and peer review Not commissioned; externally peer reviewed.

Data availability statement No data are available.

Supplemental material This content has been supplied by the author(s). It has not been vetted by BMJ Publishing Group Limited (BMJ) and may not have been peer-reviewed. Any opinions or recommendations discussed are solely those of the author(s) and are not endorsed by BMJ. BMJ disclaims all liability and responsibility arising from any reliance placed on the content. Where the content includes any translated material, BMJ does not warrant the accuracy and reliability of the translations (including but not limited to local regulations, clinical guidelines, terminology, drug names and drug dosages), and is not responsible for any error and/or omissions arising from translation and adaptation or otherwise.

Open access This is an open access article distributed in accordance with the Creative Commons Attribution Non Commercial (CC BY-NC 4.0) license, which permits others to distribute, remix, adapt, build upon this work non-commercially, and license their derivative works on different terms, provided the original work is properly cited, appropriate credit is given, any changes made indicated, and the use is non-commercial. See: http://creativecommons.org/licenses/by-nc/4.0/.

\section{ORCID iD}

Ingrid Marie Taxt Horne http://orcid.org/0000-0002-2182-4266

\section{REFERENCES}

1 Wallace JE, Lemaire JB, Ghali WA. Physician wellness: a missing quality indicator. Lancet 2009;374:1714-21.

2 Panagioti M, Geraghty K, Johnson J. Association between physician burnout and patient safety, professionalism, and patient satisfaction: a systematic review and meta-analysis. JAMA Intern Med 2018;178:1317-31.

3 Casalino LP, Crosson FJ. Physician satisfaction and physician wellbeing: should anyone care? Professions and Professionalism 2015;5.

4 Haas JS, Cook EF, Puopolo AL, et al. Is the professional satisfaction of general internists associated with patient satisfaction? J Gen Intern Med 2000;15:122-8.

5 Gustafsson S, Løvseth L, Schenck-Gustafsson K, et al. What makes physicians go to work while sick: a comparative study of sickness presenteeism in four European countries (HOUPE). Swiss Medical Weekly2013;143.

6 Davidson SK, Schattner PL. Doctors' health-seeking behaviour: a questionnaire survey. Med J Aust 2003;179:302-5.

7 BMA. Counselling and peer support services. Available: https://www. bma.org.uk/advice-and-support/your-wellbeing/wellbeing-supportservices/counselling-and-peer-support-for-doctors-and-medicalstudents [Accessed 30 Sep 2020].
8 Kollegialt Netværk for Læger. Available: https://www.laeger.dk/ kollegialt-netvaerk-for-laeger [Accessed 30 Sep 2020].

9 Shapiro J, Whittemore A, Tsen LC. Instituting a culture of professionalism: the establishment of a center for professionalism and peer support. The Joint Commission Journal on Quality and Patient Safety 2014;40-168-AP1.

$10 \mathrm{Rø} \mathrm{KEl}$, Gude T, Aasland OG. Does a self-referral counselling program reach doctors in need of help? A comparison with the general Norwegian doctor workforce. BMC Public Health 2007;7:36.

$11 \mathrm{R} ø \mathrm{KI}$, Aasland OG. Peer counsellors' views on the collegial support scheme for doctors. Tidsskrift for Den norske legeforening 2016.

12 Parsa-Parsi RW. The Revised Declaration of Geneva: A Modern-Day Physician's Pledge. JAMA 2017;318:1971-2.

13 Hertzberg TK, Skirbekk H, Tyssen R. The good doctor-strong and persevering. Tidsskrift for den Norske laegeforening 2016;136:1631-4.

14 McKevitt C, Morgan M, Dundas R, et al. Sickness absence and 'working through' illness: a comparison of two professional groups. J Public Health 1997:19:295-300.

15 Kivimäki Met al. Sickness absence in hospital physicians: 2 year follow up study on determinants. Occup Environ Med 2001;58:361-6.

16 Diderichsen S. It's just a job: a new generation of physicians dealing with career and work ideals. University of Umeå, 2017.

17 Dyrbye LN, Sotile W, Boone S, et al. A survey of US physicians and their partners regarding the impact of Work-Home conflict. J Gen Intern Med 2014;29:155-61.

18 Fuß I, Nübling M, Hasselhorn $\mathrm{H}-\mathrm{M}$, et al. Working conditions and work-family conflict in German hospital physicians: psychosocial and organisational predictors and consequences. BMC Public Health 2008;8:353.

19 Rich A, Viney R, Needleman S, et al. 'You can't be a person and a doctor': the work-life balance of doctors in training - a qualitative study. BMJ Open 2016;6:e013897.

20 Dyrbye LN, Shanafelt TD, Gill PR. Effect of a professional coaching intervention on the well-being and distress of physicians: a pilot randomized clinical trial. JAMA Internal Medicine 2019;179:1406-14.

21 Shanafelt TD, Makowski MS, Wang H, et al. Association of burnout, professional fulfillment, and self-care practices of physician leaders with their independently Rated leadership effectiveness. JAMA Netw Open 2020:3:e207961

22 West CP, Dyrbye LN, Rabatin JT, et al. Intervention to promote physician well-being, job satisfaction, and professionalism. JAMA Intern Med 2014;174:527-33.

23 Fimland SK, Kjenås AS, Rø KI. Medical students' attitudes and expectations for future working conditions. Tidsskrift for den Norske laegeforening 2019;139.

24 Network DS. Doctors' Support Network peer support for doctors and medical students with mental health concerns 2020. Available: https://www.dsn.org.uk/support-for-doctors [Accessed 3 Dec 2020].

25 Legeforeningen. Støttekollegaordningen [Peer support service], 2018. Available: https://www.legeforeningen.no/om-oss/helse-ogomsorgstilbud-for-alle-leger-og-medisinstudenter/stottekollegaord ningen/ [Accessed 3 Mar 2020].

26 PRI. Peer support. Available: https://www.pri.com/how-we-help/newyork-physicians-peer-support-group-for-malpractice-litigation-I-pr [Accessed 03 Dec 2020].

27 Garelick Al, Gross SR, Richardson I, et al. Which doctors and with what problems contact a specialist service for doctors? A cross sectional investigation. BMC Med 2007;5:26.

28 Patton. Qualitative research and evaluation methods. 4th edn. Sage Publications, 2014

29 Ressurssenteret Villa Sana [The Resource center Villa Sana] www.modum-bad.no/: Modum Bad; [cited 2021 21.5]. Available: https://www.modum-bad.no/dagtilbud/ressurssenteret-villa-sana/ [Accessed 21 May 2021].

30 Norwegian Medical Association. Helse- og omsorgstilbud for alle leger og medisinstudenter [Health and well-beeing services for all doctors and medical students]. Available: https://www. legeforeningen.no/om-oss/helse-og-omsorgstilbud-for-alle-leger-ogmedisinstudenter/[Accessed 21 May 2021].

31 Malterud K. Kvalitative forskningsmetoder for medisin og helsefag [Qualitative methods in medical research. 4th edn. Oslo: Universitetsforl, 2017.

32 Huberman A, Saldana J, Miles M. Qualitative data analysis: a methods Sourcebook. Thousand Oaks: SAGE Publications Inc. United States, 2015.

33 Shanafelt TD, Schein E, Minor LB, et al. Healing the professional culture of medicine. Mayo Clin Proc 2019;94:1556-66.

34 Wenger E. Communities of practice: learning, meaning, and identity. Cambridge university press, 1999. 
35 Fox F, Harris M, Taylor G, et al. What happens when doctors are patients? Qualitative study of GPs. Br J Gen Pract 2009;59:811-8.

36 George S, Hanson J, Jackson JL, Physician JJL. Physician, heal thyself: a qualitative study of physician health behaviors. Acad Psychiatry 2014;38:19-25.

37 Isaksson Rø K, Veggeland F, Aasland OG. Peer counselling for doctors in Norway: a qualitative study of the relationship between support and surveillance. Soc Sci Med 2016;162:193-200.

38 Braquehais MD, Arrizabalaga P, Lusilla P, et al. Gender differences in demographic and clinical features of physicians admitted to a program for medical professionals with mental disorders. Front Psychiatry 2016;7:181.

39 McGovern MP, Angres DH, Leon S. Characteristics of physicians presenting for assessment at a behavioral health center. $J$ Addict Dis 2000;19:59-73.
40 Giæver F, Lohmann-Lafrenz S, Løvseth LT. Why hospital physicians attend work while ill? The spiralling effect of positive and negative factors. BMC Health Serv Res 2016;16:548.

41 Ikeda R, Pelton C. Diversion programs for impaired physicians. West J Med 1990;152:617.

42 Rosvold EO, Bjertness E. Illness behaviour among Norwegian physicians. Scand J Public Health 2002;30:125-32.

43 Gold KJ, Goldman EB, Kamil LH, et al. No appointment necessary? Ethical challenges in treating friends and family. N Engl J Med 2014;371:1254-8.

44 Reith TP. Burnout in United States healthcare professionals: a narrative review. Cureus 2018;10:e3681.

45 Dyrbye LN, West CP, Sinsky CA, et al. Medical licensure questions and physician reluctance to seek care for mental health conditions. Mayo Clinic Proceedings 2017;92:1486-93. 